\title{
Experimental model of mild diabetes: long-term evaluation of glycemic profile and gastric contractility in rats
}

\author{
Andrieli Taise Hauschildt", Viviane Batista Behenck, Maysa Bruno de Lima, Denize Jussara Rupolo Dall'Agnol, \\ Gustavo Tadeu Volpato, Madileine Francely Américo
}

From 20th Brazilian Diabetes Society Congress

Porto Alegre, Brazil. 11-18 November 2015

\section{Background}

Diabetes mellitus is characterized by hyperglycemia resulting from progressive defects in insulin secretion and/or insulin resistance. Diabetes has been associated with several gastrointestinal complications; however, appropriate experimental models are still under study.

\section{Objective}

The aim was to evaluate gastric contractility at three and six months after the induction of mild diabetes in rats.

\section{Materials and methods}

Male Wistar rats were divided into mild diabetes $(\mathrm{n}=14)$ and control $(\mathrm{n}=8)$ groups. For mild diabetes induction, newborn rats received streptozotocin (STZ-100 mg/kg, subcutaneously) in a citrate buffer and control animals received only citrate buffer on the first day of life. The oral glucose tolerance test (OGTT) was performed three months later and blood glucose was measured monthly by glucometer Fácil True Read (Home Diagnostics ${ }^{\circledR}$ ). The gastric contractility was evaluated by Alternate Current Biosusceptometry (ACB), a previously validated and noninvasive biomagnetic technique. After $12 \mathrm{~h}$ fasting, the animals were fed with $2 \mathrm{~g}$ of magnetically marked chow and anesthetized with sodium pentobarbital (40 mg/kg, intraperitoneally). All rats were placed in a supine position with ACB sensor positioned on the gastric region during $30 \mathrm{~min}$. Frequency and amplitude of gastric contractions, also abnormal rhythmic index, were obtained from signals. Comparisons were performed by ANOVA (Tukey), being significant at $\mathrm{p}<0.05$. OGTT

* Correspondence: hauschildt.biomed@gmail.com

Universidade Federal de Mato Grosso, Barra do Garças, Brazil confirmed diabetes in the STZ-induced group, demonstrating the success of the model.

\section{Results}

Mild blood glucose levels were observed in STZ-induced group at $3 \mathrm{rd}(155.8 \pm 32.3 \mathrm{mg} / \mathrm{dL})$ and 6th months $(134.3 \pm 9.5 \mathrm{mg} / \mathrm{dL})$ compared to control $(97.0 \pm 10.9 \mathrm{mg} / \mathrm{dL})$. There was no significant difference in frequency of gastric contraction between groups. In contrast, the amplitude of contractions were stronger in moderately diabetic rats after three $(0.26 \pm 0.13$ Volts $/ \mathrm{s})$ and six months $(2.22 \pm 0.96$ Volts/s) compared to control $(0.018 \pm 0.07 \mathrm{Volts} / \mathrm{s})$, and this change was more pronounced at 6 th month than in the 3rd month. Abnormalities in the stomach contractions rhythm during the control (9.2 $\pm 5.3 \%)$, 3rd (46.4 $48.1 \%)$ and 6th months $(39.0 \pm 13.3 \%)$ post-induction corroborate these motor disorders temporally associated with diabetes.

\section{Conclusions}

Moderately elevated blood glucose levels over a long period may be related to the gastrointestinal complications since the gastric contractility is more impaired in the sixth than third month, by a mechanism that may be associated with neuropathy.

Published: 11 November 2015

doi:10.1186/1758-5996-7-S1-A10

Cite this article as: Hauschildt et al:: Experimental model of mild diabetes: long-term evaluation of glycemic profile and gastric contractility in rats. Diabetology \& Metabolic Syndrome 2015 7(Suppl 1): A10. 